\title{
Chronisch entzündliche Darmerkrankungen durch zu viel Antibiotika?
}

\author{
Nach den Ergebnissen einer kana- \\ dischen Fall-Kontroll-Studie erhöhen \\ wiederholte Antibiotika-Behand- \\ lungen das Risiko für chronisch ent- \\ zündliche Darmerkrankungen um \\ $50 \%$.
}

- Die chronisch entzündlichen Darmkrankheiten (CED) wie Morbus Crohn (MC) und Colitis ulcerosa (CU) entstehen wahrscheinlich als Folge einer Interaktion zwischen einer genetischen Prädisposition für eine ausgiebige immunologische Antwort und einer Antwort auf Umweltfaktoren, vor allem Darmbakterien. Antibiotika verändern die Darmflora: Die mikrobielle Besiedlung wird vermindert, bestimmte Bakterien sind davon besonders betroffen.

Die Universität von Manitoba verfügt über eine Sammlung epidemiologischer Daten von CED-Patienten. Der Antibiotikaverbrauch von 2234 Patienten mit CED wurde verglichen mit dem von 22346 Kontrollpersonen.

Das Durchschnittsalter der betrachteten Patienten war 43 Jahre. 56\% der CED-Patienten sowie 66\% der Kontrollpatienten hatten in den zwei Jahren vor

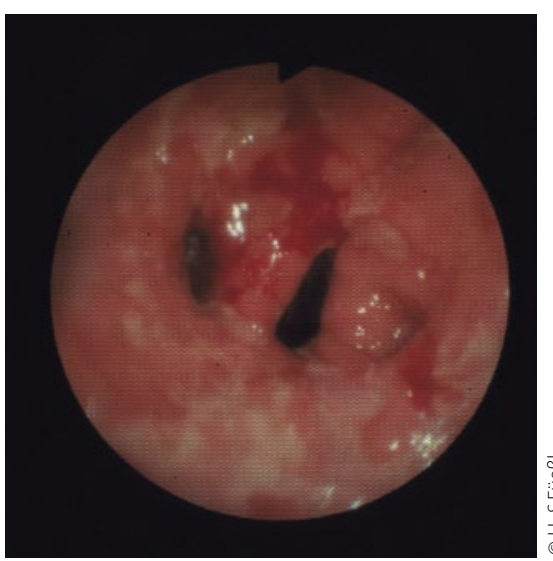

Morbus Crohn: bei wiederholter Antibiotikagabe häufiger.

Diagnosestellung keine antibiotische Behandlung erhalten. 12\% der CED-Patienten, dies entspricht 262 Fällen, hatten mehr als drei antibiotische Behandlungen in den beiden Jahren vor der Diagnose im Vergleich zu 7\% $(n=1.527)$ der Kontrollpersonen.

Ähnliche Unterschiede zwischen Kontrollpersonen und Patienten ergaben sich in den drei, vier und fünf Jahren vor der Diagnose CED. Das Risiko, an einer CED zu erkranken, stieg durch wiederholte Antibiotikabehandlung auf das 1,5-fache an. Dies gilt sowohl für MC als auch CU. Bei mehr als drei Antibiotikabehandlungen ist das Risiko für $\mathrm{CU}$ höher als für MC. Der Einfluss der Antibiotikabehandlung auf die Entstehung von CED betraf alle Altersgruppen.

\section{Kommentar}

In dieser populationsbasierten Analyse ist eine mäßig ausgeprägte, aber konstant durch alle Altergruppen nachweisbare Assoziation zwischen Antibiotikagebrauch und dem Auftreten von CED nachweisbar. Ob es sich dabei um einen kausalen Mechanismus handelt, bleibt offen. Die Forderung nach einem sparsamen Umgang mit Antibiotika ist ernst zu nehmen, auch wenn weitergehende Untersuchungen über einen Zusammenhang von Darmflora und stattgehabten Antibiotika-Behandlungen im Zusammenhang mit der Entstehung von chronisch entzündlichen Darmkrankheiten fehlen.

R. BÜCHSEL =

- S. Y. Shaw, J. F. Blanchard, C. N. Bernstein Association between the Use of Antibiotics and New Diagnoses of Crohn's Disease and Ulcerative Colitis. Am. J. Gastroenterol. 106 (2011) 2133-2142

\section{Immunisierung gegen Alzheimer in Sicht?}

Bei der Suche nach einer Heilbehandlung für die Alzheimerdemenz werden verschiedene Ansätze verfolgt. Eine Vakzine mit Antikörpern gegen das Betaamyloid in den Alzheimerplaques gilt als besonders vielversprechend. Allerdings hatten die ersten Vakzinen zu viele und zu gravierende Nebenwirkungen.

- Wissenschaftler des Karolinska Institutes in Stockholm haben jetzt eine aktive Immunisierung mit Eigen- $\beta$-Amyloid erprobt. Dabei wurde die Vakzine so modifiziert, dass sie nur noch Reaktionen ge- gen das schädigende $B$-Amyloid auslöste. Die alte Vakzine hatte T-Zellen aktiviert, die das Gehirn angriffen.

Mit der neuen Vakzine entwickelten 80\% der Patienten schützende Antikörper gegen $\beta$-Amyloid ohne ernsthafte Nebenwirkungen. Die Forscher meinen, dass ihre AD106-Vakzine eine tolerierbare Behandlung der Patienten mit schwachem oder mäßigem Alzheimer zulässt.

\section{Kommentar}

Diese Studie sollte vor allem die Sicherheit des neuen Impfstoffes prüfen. Nachgewiesen wurde auch eine Immunreaktion gegen $\beta$-Amyloid. Ob dies jetzt der Durchbruch bei der Alzheimerdemenz ist, müssen weitere Studien zeigen.

K. MALBERG =

\footnotetext{
- B. Winblad et al.

Safety, tolerability, and antibody response of active $A-\beta$ immunotherapy with CAD106 in patients with Alzheimer's disease: randomised, double-blind, placebo-controlled, first-inhuman study. Early online published on $06 \mathrm{Ju}-$ ne 2012 iThe Lancet Neurology; doi: 10.1016/ S1474-4422(12)70140-0
} 